\title{
JACIE Accreditation of HSCT Programs
}

\author{
Riccardo Saccardi, Eoin McGrath, \\ and John A. Snowden
}

\subsection{Introduction}

The complexity of HSCT as a medical technology and the frequent need for close interaction and interdependence between different services, teams, and external providers (donor registries, typing laboratories, etc.) distinguish it from many other medical fields. Approximately 20 years ago, this complexity led to efforts by transplantation professionals to standardize processes based on consensus as a way to better manage inherent risks of this treatment. HSCT was, and continues to be, a pioneer in the area of quality and standards.

\footnotetext{
R. Saccardi $(\bowtie)$

Department of Cellular Therapies and Transfusion

Medicine, Careggi University Hospital,

Florence, Italy

e-mail: riccardo.saccardi@unifi.it

E. McGrath

European Society for Blood and Marrow

Transplantation (EBMT), Barcelona, Spain

J. A. Snowden

Department of Haematology, Sheffield Teaching

Hospitals NHS Foundation Trust, University

of Sheffield, Sheffield, UK
}

\subsection{Background}

In 1998, EBMT and the International Society for Cell \& Gene Therapy (ISCT) established the Joint Accreditation Committee, ISCT and EBMT (JACIE), aimed to offer an inspection-based accreditation process in HSCT against established international standards. JACIE is a committee of the EBMT, its members are appointed by and are accountable to the EBMT Board, and ISCT is represented through two members of the Committee. JACIE collaborates with the US-based Foundation for the Accreditation of Cellular Therapy (FACT) to develop and maintain global standards for the provision of quality medical and laboratory practice in cellular therapy.

The JACIE and FACT accreditation systems stand out as examples of profession-driven initiatives to improve quality in transplantation and which have subsequently been incorporated by third parties, such as healthcare payers (health insurers, social security) and competent authorities (treatment authorization). The JACIE Accreditation Program was supported in 2004 by the European Commission under the public health program 2003-2008 and was acknowledged as an exemplary project in a 2011 review of spending under the public health program. 


\subsection{Impact of Accreditation in Clinical Practice}

Much literature indicating a better clinical outcome in teaching hospitals and centers of excellence has been available since the 1990s (Hartz et al. 1989; Birkmeyer et al. 2005; Loberiza et al. 2005). Initial evidence of a positive relationship between the implementation of a quality management system and outcome of HSCT in Europe was published in 2011 (Gratwohl et al. 2011). In this paper, patients' outcome was systematically better when the transplantation center was at a more advanced phase of JACIE accreditation, independent of year of transplantation and other risk factors.

Another analysis (Gratwohl et al. 2014) was performed on a large cohort of patients who received either an allogeneic or an autologous transplantation between 1996 and 2006 and reported to the EBMT database. The authors showed that the decrease of overall mortality in allogeneic procedures over the 14-year observation period was significantly faster in JACIEaccredited centers, thus resulting in a higher relapse-free survival and overall survival at 72 months from transplant. Such improvement was not shown in autologous transplantation.

Similar results published by Marmor et al. (2015) in an American study showed that centers accredited by both FACT and Clinical Trial Network (CTN) demonstrated significantly better results for more complex HSCT such as HLAmismatched transplants.

These data reinforce the concept that clinical improvement is driven by the implementation of a quality management system embedded in external accreditation standards, especially in the context of more complex procedures. This process also results in a wider standardization of procedures across different countries and geographic areas, therefore contributing to providing patients with similar treatment expectations even when accessing different health management systems. A comprehensive review of this was recently published (Snowden et al. 2017).

\subsection{JACIE-FACT Accreditation System}

JACIE and FACT accreditation systems are based on the development and continuous update of standards covering the entire transplantation process, from selection of the donor/patient to follow-up, including collection, characterization, processing, and storage of the graft. Considering the different competences included in the process, the standards are articulated in four parts:

- Clinical Program,

- Bone Marrow Collection,

- Apheresis Collection and

- Processing Facility.

A quality management (QM) section is embedded in each section, aimed at providing a tool for both the applicants to develop a comprehensive system of quality assessment and for the inspectors to check the compliance of the program to the standard. Stand-alone processing labs can apply; however, the target of the accreditation is the transplantation program, intended as a process in its entirety, thus requiring a full integration of units, laboratories, services, and professionals. Each section focusses on the competence of personnel, listing topics for which the evidence of a specific training is required which also includes the minimum experience requirements for positions of responsibility. Maintaining these competences is also required for all professionals.

The standards are revised on a 3-year basis by a commission formed of experts appointed by JACIE and FACT, including HSCT administration, cell processing and storage, blood apheresis, transplant registries, and QM specialists. The standards are based on published evidence and, when this is not available, on expert consensus. A legal review and comparison with current regulations are carried out for each version. When the developmental phase is finalized, the standards are published for public review and comment and finally approved by JACIE and FACT. 
The standards incorporate sound principles of quality medical and laboratory practice in cellular therapy, but do not cover legal requirements of local competent authorities.

The compliance to the standards is ensured through an inspection system, carried out by voluntary inspectors, trained and coordinated by the JACIE office in Barcelona. The JACIE inspection is a multistep procedure: the applicant center is provided with all the application documents and is then required to submit a set of documentation to the JACIE accreditation coordinators. If the first review is positive, the on-site inspection is then planned in agreement with the applicant.

JACIE inspections are carried out in most cases in the language of the applicant. The inspectors' report is then assessed by the JACIE accreditation committee, which may request supplementary information, modifications, or another on-site visit. If all aspects are shown to be compliant, accreditation is awarded. An accreditation cycle is 4 years for JACIE, and facilities must complete an interim desk-based audit after 2 years post-accreditation. Accredited facilities must reapply for reaccreditation and may also be reinspected in response to complaints or information that a facility may be noncompliant with the standards, in response to significant changes in the program and/or facility or as determined by JACIE.

Many tools are made available to prepare the accreditation through the JACIE website, including a quality management guide, the welcome guide, and webinars. JACIE runs training courses throughout the year, and the Barcelonabased staff are available to support the applicants. An accreditation manual provides detailed explanations and examples for each single item of the standards. A special approach is under development for transplant programs in lowand middle-income countries (LMICs), where full accreditation might not be feasible due to resources and/or cultural issues. In this case, a stepped process toward accreditation is being developed, based on the selection of organizational items of the standard which may be ful- filled by the implementation of a QM system, without requiring specific investments in infrastructures and/or equipment. This "stepwise" option will also encourage the programs to connect with an international network of professionals and may also stimulate local authorities to support further progress toward full accreditation in the interests of patients, donors, and the professional community.

The standards cover the use of different sources of hematopoietic stem cells and nucleated cells from any hematopoietic tissue source administered in the context of the transplant process, such as DLI. The term "hematopoietic" in the title is to define the scope of these standards, due to an increasing number of accredited facilities that also support non-hematopoietic cellular therapies. Starting with version 6.1, the standards include new items specifically developed for other cellular therapy products, with special reference to immune effector cells (IECs). This reflects the rapidly evolving field of cellular therapy through mainly, but not exclusively, genetically modified cells, such as CAR-T cells. The standards do not cover the manufacturing of such cells but include the chain of responsibilities where the product is provided by a third party and ensure the competence of the personnel in the management of adverse events related to the infusion.

Another recent development has been the introduction of "benchmarking" standards related to 1-year survival and other patient outcomes. If center performance is below the expected range, then a corrective action plan is mandated. The requirement for a risk-adapted "benchmarking" system is being addressed in the development of the new EBMT MACRO registry, which will enable centers to address these new JACIE standards within their own BMT community and across international boundaries.

JACIE is run on a non-profit basis, resourced almost entirely on application fees. Fees depend on the configuration of the program and its EBMT membership status. At the time of writing in February 2018, the application fee for a transplant 
program made up of collection, processing, and clinical units is $€ 14,600$ for EBMT members and $€ 29,200$ for non-EBMT members. Supplementary fees for additional sites and discounts for active inspectors in the team are applied (see JACIE website for details).

Overall, over 600 accreditation inspections have been carried out in 25 countries, representing over $40 \%$ transplant centers in Europe
(Figs. 5.1 and 5.2), many of which have been through more than one accreditation cycle. JACIE accreditation is now mandatory in several European countries, to apply for reimbursement of the procedure and/or to be authorized to perform HSCT. JACIE also represents an opportunity for centers in LMICs to align their organizations with practice in the more advanced HSCT programs.

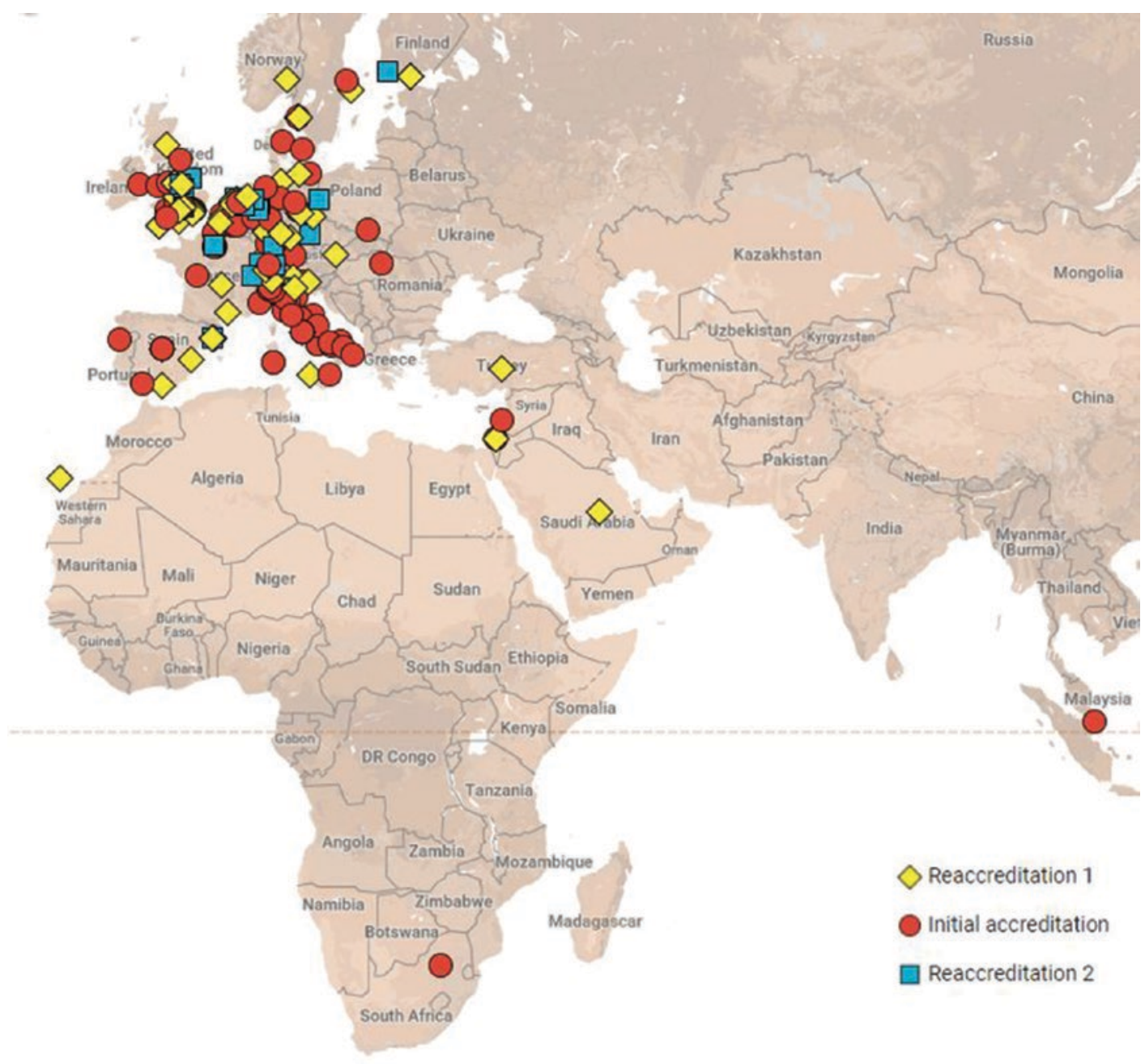

Fig. 5.1 JACIE-accredited programs March 2018 


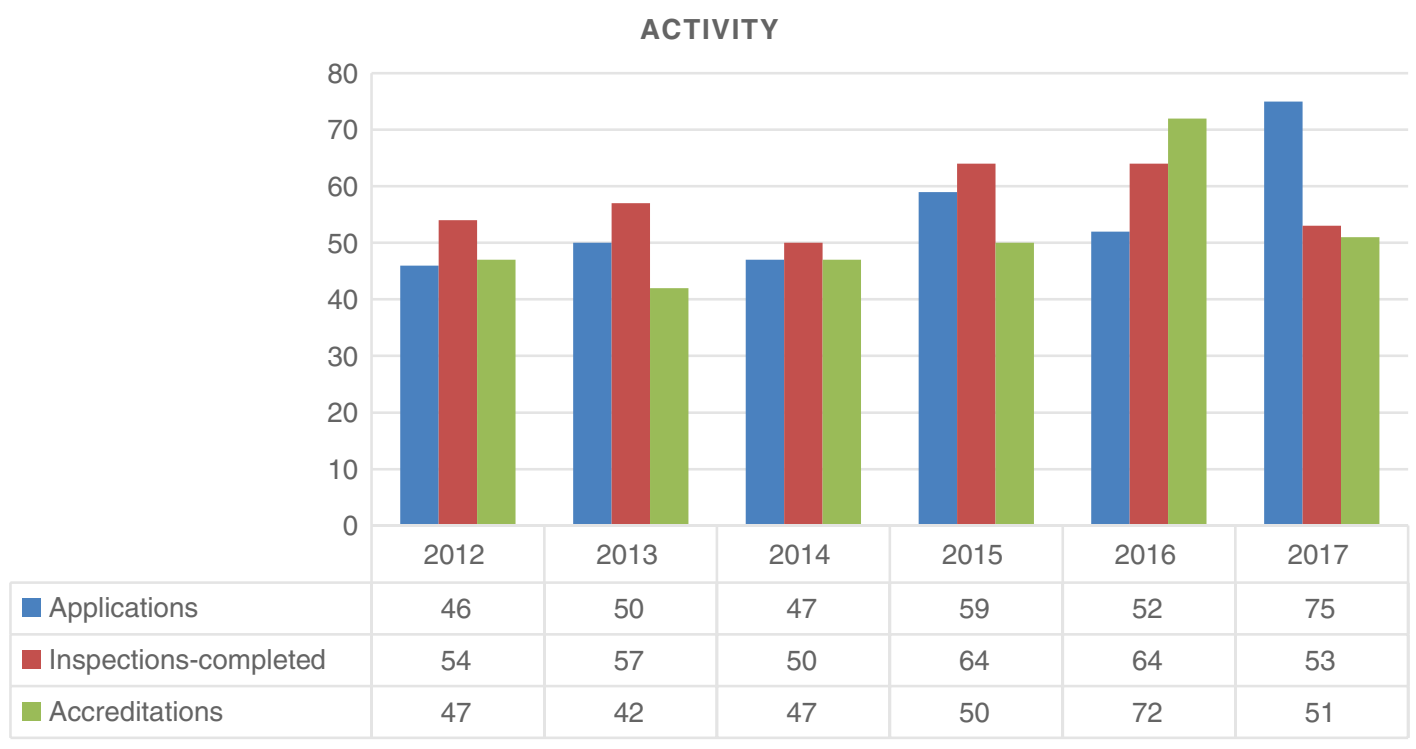

Fig. 5.2 JACIE activity 2012-2017

\section{Key Points}

- JACIE accreditation is based on an internationally agreed quality standard system led and delivered by HSCT and cell therapy professionals.

- The standards are regularly updated, incorporating advances in the evidence base while reflecting the practical view of experienced experts on clinical and laboratory practice of HSCT and cell therapy.

- Published data support a positive improvement in the clinical outcome related to the accreditation process, also promoting a progressive standardization of HSCT practice across different countries.

- Recent developments in the standards include development of standards for CAR-T and other immune effector cells (IEC), "benchmarking" of patient survival and access of centers in LMIC to the "stepwise" accreditation.

\section{References}

Birkmeyer NJ, Goodney PP, Stukel TA, et al. Do cancer centers designated by the National Cancer Institute have better surgical outcomes? Cancer. 2005;103:435-41.

Gratwohl A, Brand R, McGrath E, Joint Accreditation Committee of the International Society for Cellular, B. the European Group for, T. Marrow and N. the European Leukemia, et al. Use of the quality management system "JACIE" and outcome after hematopoietic stem cell transplantation. Haematologica. 2014;99:908-15.

Gratwohl A, Brand R, Niederwieser D, et al. Introduction of a quality management system and outcome after hematopoietic stem-cell transplantation. J Clin Oncol. 2011;29:1980-6.

Hartz AJ, Krakauer H, Kuhn EM, et al. Hospital characteristics and mortality rates. $\mathrm{N}$ Engl $\mathrm{J}$ Med. 1989;321:1720-5.

Loberiza FR, Zhang MJ, Lee SJ, et al. Association of transplant center and physician factors on mortality after hematopoietic stem cell transplantation in the United States. Blood. 2005;105:2979-87.

Marmor S, Begun JW, Abraham J, Virnig BA. The impact of center accreditation on hematopoietic cell transplantation (HCT). Bone Marrow Transplant. 2015;50:87-94.

Snowden JA, McGrath E, Duarte RF, et al. JACIE accreditation for blood and marrow transplantation: past, present and future directions of an international model for healthcare quality improvement. Bone Marrow Transplant. 2017;52:1367-71. 
Open Access This chapter is licensed under the terms of the Creative Commons Attribution 4.0 International License (http://creativecommons.org/licenses/by/4.0/), which permits use, sharing, adaptation, distribution and reproduction in any medium or format, as long as you give appropriate credit to the original author(s) and the source, provide a link to the Creative Commons license and indicate if changes were made.

The images or other third party material in this chapter are included in the chapter's Creative Commons license, unless indicated otherwise in a credit line to the material. If material is not included in the chapter's Creative Commons license and your intended use is not permitted by statutory regulation or exceeds the permitted use, you will need to obtain permission directly from the copyright holder.

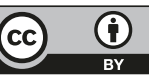

